\title{
Why hot water freezes faster than cold?
}

\author{
Lutvo Kurić \\ Institute of Economics, University of Sarajevo, Trg Oslobođenja 1, Sarajevo, \\ Bosnia and Herzegovina \\ E-mail address: lutvokuric@yahoo.com
}

\begin{abstract}
The modern science mainly treats the biochemical basis of sequencing in bio-macromolecules and processes in chemistry and biochemistry. In these informational cybernetic principles we seek to answer the following question: Why hot water freezes faster than cold? These principles determine the freezing point of water. Determine that the hot water freezes faster than cold. What we did is the following: We translated the physical and chemical parameters from the language of water into the digital language of programmatic, cybernetic and information principles. This we did by using the adequate mathematical algorithms. By using chemical-information procedures, we calculated the numerical value for the information content of molecule of water. What we got this way is the digital picture of this molecule. These digital pictures reveal to us a whole new dimension of water. They reveal to us that the chemical process of this molecule is strictly conditioned and determined by programmatic, cybernetic and information principles.
\end{abstract}

Keywords: Water; Hot Water; Freezing hot water; Freezing cold water

\section{INTRODUCTION}

In these informational cybernetic principles we seek to answer the following question: Why hot water freezes faster than cold?

These principles determine the freezing point of water. Determine that the hot water freezes faster than cold. What we did is the following: We translated the physical and chemical parameters from the language of water into the digital language of programmatic, cybernetic and information principles. This we did by using the adequate mathematical algorithms.

By using chemical-information procedures, we calculated the numerical value for the information content of molecule of water.

What we got this way is the digital picture of this molecule. These digital pictures reveal to us a whole new dimension of water. They reveal to us that the chemical process of this molecule is strictly conditioned and determined by programmatic, cybernetic and information principles. 


\section{RESULTS}

Digital image where chemistry is represented is in the form of numbers. That image can be created with the help of the new scientific methods. At the first stage of our research we replaced the chemical elements from the chemical formulae with atomic numbers, numbers of atoms, atomic weight and other numerical values in those formulas.

By this way we got digital image of chemistry. Then we mathematically analyzed those digital images. After we making such analysis, we discovered existence of digital codes in those images, which interconnect all chemical elements and other sequences in chemistry. Given below is a brief introduction about the way we discovered those chemistry digital codes and how those codes interconnect all chemical elements in this science.

\section{1. Dissociation constant of water}

As a frequently used special case, the dissociation constant of water is often expressed as $K_{\mathrm{w}}$ : The concentration of water is not included in the definition of $K_{\mathrm{w}}$, for reasons described in the article equilibrium constant.

The value of $K_{\mathrm{w}}$ varies with temperature, as shown in the table below. This variation must be tak en into account when making precise measurements of quantities such as $\mathrm{pH}$.

Why hot water freezes faster than cold?

\begin{tabular}{|c|c|c|}
\hline Water temperature & $\mathbf{K}_{\mathbf{w}} / \mathbf{1 0}^{-\mathbf{1 4}}$ & $\mathbf{p K}_{\mathbf{w}}$ \\
\hline $0{ }^{\circ} \mathrm{C}$ & 0.1 & 14.92 \\
\hline $10^{\circ} \mathrm{C}$ & 0.3 & 14.52 \\
\hline $18^{\circ} \mathrm{C}$ & 0.7 & 14.16 \\
\hline $25^{\circ} \mathrm{C}$ & 1.2 & 13.92 \\
\hline $30^{\circ} \mathrm{C}$ & 1.8 & 13.75 \\
\hline $50^{\circ} \mathrm{C}$ & 8.0 & 13,10 \\
\hline $60^{\circ} \mathrm{C}$ & 12.6 & 12.90 \\
\hline $70^{\circ} \mathrm{C}$ & 21.2 & 12.67 \\
\hline $80^{\circ} \mathrm{C}$ & 35 & 12.46 \\
\hline $90^{\circ} \mathrm{C}$ & 53 & 12.28 \\
\hline $100^{\circ} \mathrm{C}$ & 73 & 12.14 \\
\hline
\end{tabular}

\section{2. Digital language of dissociation constant of water}

The matrix mechanism of water temperature have been analyzed by the application of cybernetic methods, information theory and system theory, respectively. First, we decode the dissociation constant of water expressed as $\mathrm{pK}_{\mathrm{w}}$ : 


\section{Example 1.}

\begin{tabular}{|c|c|c|}
\hline Water temperature & $\mathrm{K}_{\mathrm{W}} / 10^{-14}$ & $\mathrm{pK}_{\mathrm{w}}$ \\
\hline $0^{\circ} \mathrm{C}$ & 0.1 & 14.92 \\
\hline $100^{\circ} \mathrm{C}$ & 73 & 12.14 \\
\hline Sum & & $\mathbf{2 7 , 0 6}$ \\
\hline
\end{tabular}

\begin{tabular}{|c|c|c|}
\hline Water temperature & $\mathrm{K}_{\mathrm{w}} / 10^{-14}$ & $\mathrm{pK}_{\mathrm{w}}$ \\
\hline $18^{\circ} \mathrm{C}$ & 0.7 & 14.16 \\
\hline $60^{\circ} \mathrm{C}$ & 12.6 & 12.90 \\
\hline Sum & & $\mathbf{2 7 , 0 6}$ \\
\hline
\end{tabular}

$[(0+100)-(60+18)]=22 ;(27,06 \times 100)=(22+22+22 \ldots+22) ;$

\section{Determinate 2 x 2}

$$
\begin{gathered}
\operatorname{DET}(0.1,14.92,12.14,73)=\mathbf{- 1 6 8} ; \\
\operatorname{DET}(0.7,14.16,12.6,12.90)=\mathbf{- 1 6 8} ;
\end{gathered}
$$

In this example there a mathematical balance between constants $\mathrm{K}_{\mathrm{w}} / 10^{-14}$ and $\mathrm{pK}_{\mathrm{w}}$. This balance of the determine program, cyber laws and information.

\section{Example 2.}

\begin{tabular}{|c|c|c|}
\hline Water temperature & $\mathrm{K}_{\mathrm{w}} / 10^{-14}$ & $\mathrm{pK}_{\mathrm{w}}$ \\
\hline $10^{\circ} \mathrm{C}$ & 0.3 & 14.52 \\
\hline $18^{\circ} \mathrm{C}$ & 0.7 & 14.16 \\
\hline $60^{\circ} \mathrm{C}$ & 12.6 & 12.90 \\
\hline $90^{\circ} \mathrm{C}$ & 53 & 12.28 \\
\hline Sum & & $\mathbf{5 3 , 8 6}$ \\
\hline
\end{tabular}

\begin{tabular}{|c|c|c|}
\hline Water temperature & $\mathrm{K}_{\mathrm{w}} / 10^{-14}$ & $\mathrm{pK}_{\mathrm{w}}$ \\
\hline $0^{\circ} \mathrm{C}$ & 0.1 & 14.92 \\
\hline $10^{\circ} \mathrm{C}$ & 0.3 & 14.52 \\
\hline $90^{\circ} \mathrm{C}$ & 53 & 12.28 \\
\hline $100^{\circ} \mathrm{C}$ & 73 & 12.14 \\
\hline Sum & & $\mathbf{5 3 , 8 6}$ \\
\hline
\end{tabular}


Determinate $4 \times 4$

\begin{tabular}{|c|c|c|c|}
\hline 0,3 & 14,52 & 0,7 & 14,16 \\
\hline 12,6 & 12,90 & 53 & 12,28 \\
\hline 0,1 & 14,92 & 0,3 & 14,52 \\
\hline 53 & 12,28 & 73 & 12,14 \\
\hline \multicolumn{4}{|c}{} \\
\\
$\quad$ \\
0
\end{tabular}

Input:

$\{0,3,14,52,0,7,14,16,12,6,12,90,53$,

$12,28,0,1,14,92,0,3,14,52,53,12,28,73,12,14\}$

Plot:

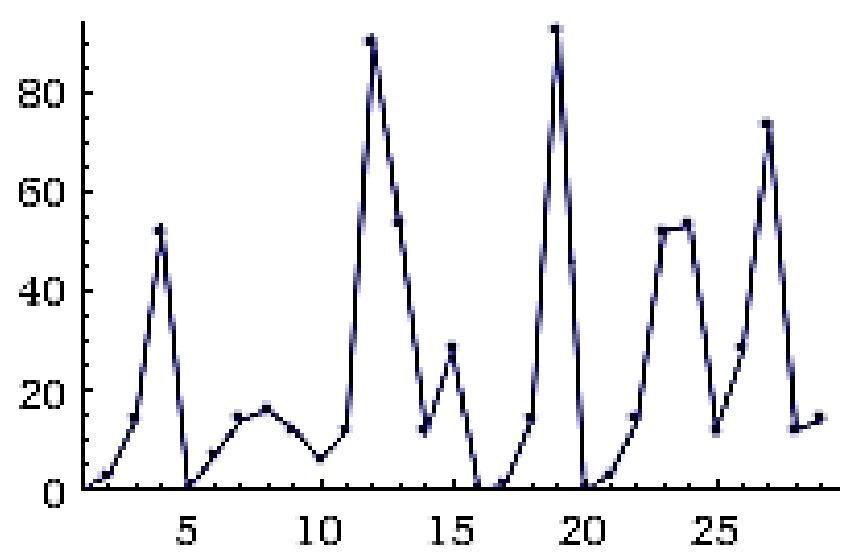

Number line:

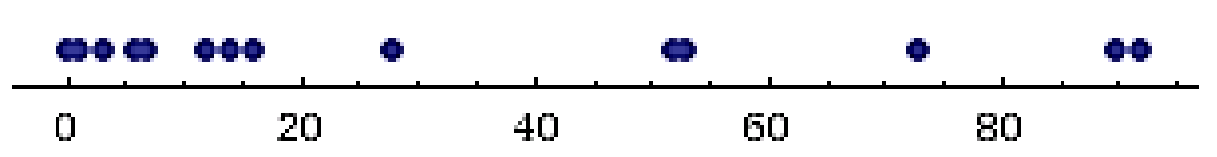

Length of data:

29 items

Total:

$$
0+3+\cdots+12+14=687
$$




\section{Statistics:}

\begin{tabular}{|l|l|}
\hline mean & 23.69 \\
\hline median & 14 \\
\hline sample standard deviation & 26.91 \\
\hline
\end{tabular}

Box-and-whisker chart:

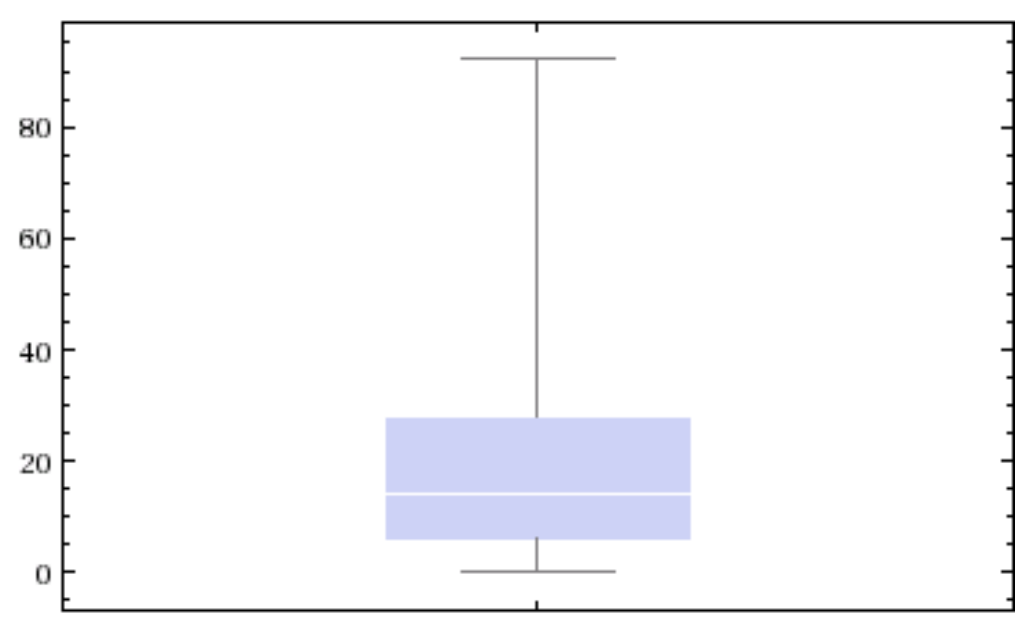

Differences:

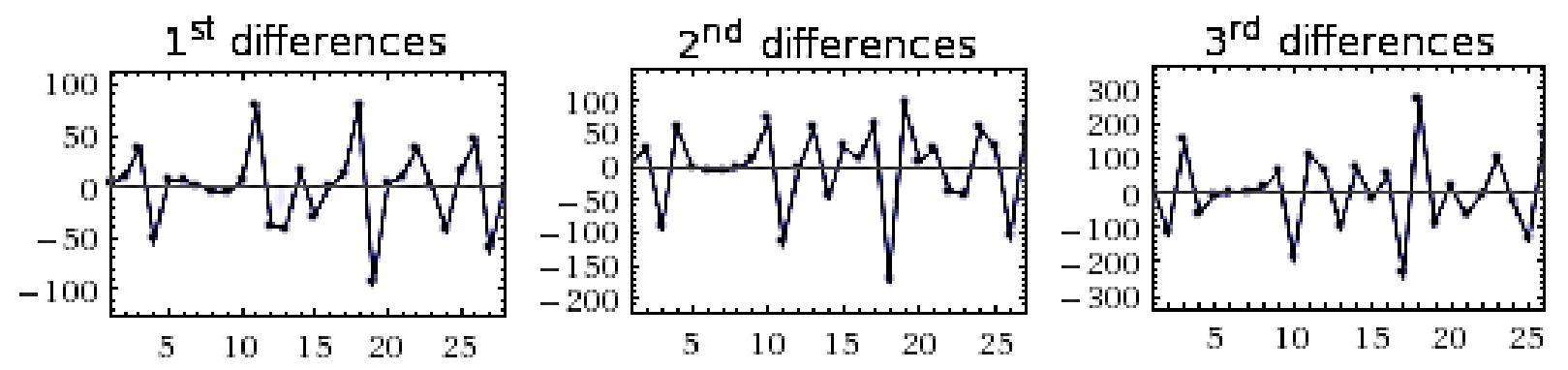

Cumulative sums:

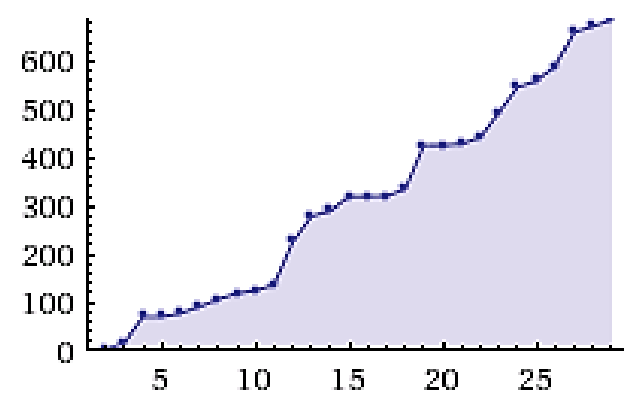


In this example there is a balance of size $\mathrm{K}_{\mathrm{w}} / 10^{-14}$ and $\mathrm{pK}$.

Example 3.

\begin{tabular}{|c|c|c|}
\hline Water temperature & $\mathrm{K}_{\mathrm{w}} / 10^{-14}$ & $\mathrm{pK}_{\mathrm{w}}$ \\
\hline $10^{\circ} \mathrm{C}$ & 0.3 & 14.52 \\
\hline $18^{\circ} \mathrm{C}$ & 0.7 & 14.16 \\
\hline $60^{\circ} \mathrm{C}$ & 12.6 & 12.90 \\
\hline $80^{\circ} \mathrm{C}$ & 35 & 12.46 \\
\hline Sum & & $\mathbf{5 4 , 0 4}$ \\
\hline
\end{tabular}

\begin{tabular}{|c|c|c|}
\hline Water temperature & $\mathrm{K}_{\mathrm{w}} / 10^{-14}$ & $\mathrm{pK}_{\mathrm{w}}$ \\
\hline $0{ }^{\circ} \mathrm{C}$ & 0.1 & 14.92 \\
\hline $10^{\circ} \mathrm{C}$ & 0.3 & 14.52 \\
\hline $80^{\circ} \mathrm{C}$ & 35 & 12.46 \\
\hline $100^{\circ} \mathrm{C}$ & 73 & 12.14 \\
\hline Sum & & $\mathbf{5 4 , 0 4}$ \\
\hline
\end{tabular}

Example 4.

\begin{tabular}{|c|c|c|}
\hline Water temperature & $\mathrm{K}_{\mathrm{w}} / 10^{-14}$ & $\mathrm{pK}_{\mathrm{w}}$ \\
\hline $18^{\circ} \mathrm{C}$ & 0.7 & 14.16 \\
\hline $25^{\circ} \mathrm{C}$ & 1.2 & 13.92 \\
\hline $30^{\circ} \mathrm{C}$ & 1.8 & 13.75 \\
\hline $50^{\circ} \mathrm{C}$ & 8.0 & 13,10 \\
\hline $60^{\circ} \mathrm{C}$ & 12.6 & 12.90 \\
\hline Sum & & $\mathbf{6 7 , 8 3}$ \\
\hline
\end{tabular}

\begin{tabular}{|c|c|c|}
\hline Water temperature & $\mathrm{K}_{\mathrm{w}} / 10^{-14}$ & $\mathrm{pK}_{\mathrm{w}}$ \\
\hline $0{ }^{\circ} \mathrm{C}$ & 0.1 & 14.92 \\
\hline $25^{\circ} \mathrm{C}$ & 1.2 & 13.92 \\
\hline $30^{\circ} \mathrm{C}$ & 1.8 & 13.75 \\
\hline $50^{\circ} \mathrm{C}$ & 8.0 & 13,10 \\
\hline $100^{\circ} \mathrm{C}$ & 73 & 12.14 \\
\hline Sum & & $\mathbf{6 7 , 8 3}$ \\
\hline
\end{tabular}




\section{Example 5.}

\begin{tabular}{|c|c|c|}
\hline Water temperature & $\mathrm{K}_{\mathrm{W}} / 10^{-14}$ & $\mathrm{pK}_{\mathrm{W}}$ \\
\hline $18^{\circ} \mathrm{C}$ & 0.7 & 14.16 \\
\hline $25^{\circ} \mathrm{C}$ & 1.2 & 13.92 \\
\hline $30^{\circ} \mathrm{C}$ & 1.8 & 13.75 \\
\hline $50^{\circ} \mathrm{C}$ & 8.0 & 13,10 \\
\hline $60^{\circ} \mathrm{C}$ & 12.6 & 12.90 \\
\hline $70^{\circ} \mathrm{C}$ & 21.2 & 12.67 \\
\hline Sum & & $\mathbf{8 0 , 5 0}$ \\
\hline
\end{tabular}

\begin{tabular}{|c|c|c|}
\hline Water temperature & $\mathrm{K}_{\mathrm{w}} / 10^{-14}$ & $\mathrm{pK}_{\mathrm{w}}$ \\
\hline $0^{\circ} \mathrm{C}$ & 0.1 & 14.92 \\
\hline $25^{\circ} \mathrm{C}$ & 1.2 & 13.92 \\
\hline $30^{\circ} \mathrm{C}$ & 1.8 & 13.75 \\
\hline $50^{\circ} \mathrm{C}$ & 8.0 & 13,10 \\
\hline $70^{\circ} \mathrm{C}$ & 21.2 & 12.67 \\
\hline $100^{\circ} \mathrm{C}$ & 73 & 12.14 \\
\hline Sum & & $\mathbf{8 0 , 5 0}$ \\
\hline
\end{tabular}

\begin{tabular}{|c|c|c|}
\hline Water temperature & $\mathrm{K}_{\mathrm{w}} / 10^{-14}$ & $\mathrm{pK}_{\mathrm{w}}$ \\
\hline $0{ }^{\circ} \mathrm{C}$ & 0.1 & 14.92 \\
\hline $18^{\circ} \mathrm{C}$ & 0.7 & 14.16 \\
\hline $25^{\circ} \mathrm{C}$ & 1.2 & 13.92 \\
\hline $60^{\circ} \mathrm{C}$ & 12.6 & 12.90 \\
\hline $80^{\circ} \mathrm{C}$ & 35 & 12.46 \\
\hline $100^{\circ} \mathrm{C}$ & 73 & 12.14 \\
\hline Sum & & $\mathbf{8 0 , 5 0}$ \\
\hline
\end{tabular}




\section{Example 6.}

\begin{tabular}{|c|c|c|}
\hline Water temperature & $\mathrm{K}_{\mathrm{W}} / 10^{-14}$ & $\mathrm{pK}_{\mathrm{w}}$ \\
\hline $18^{\circ} \mathrm{C}$ & 0.7 & 14.16 \\
\hline $25^{\circ} \mathrm{C}$ & 1.2 & 13.92 \\
\hline $30^{\circ} \mathrm{C}$ & 1.8 & 13.75 \\
\hline $50^{\circ} \mathrm{C}$ & 8.0 & 13,10 \\
\hline $60^{\circ} \mathrm{C}$ & 12.6 & 12.90 \\
\hline $70^{\circ} \mathrm{C}$ & 21.2 & 12.67 \\
\hline $80^{\circ} \mathrm{C}$ & 35 & 12.46 \\
\hline Sum & & 92,96 \\
\hline
\end{tabular}

\begin{tabular}{|c|c|c|}
\hline Water temperature & $\mathrm{K}_{\mathrm{w}} / 10^{-14}$ & $\mathrm{pK}_{\mathrm{w}}$ \\
\hline $0^{\circ} \mathrm{C}$ & 0.1 & 14.92 \\
\hline $25^{\circ} \mathrm{C}$ & 1.2 & 13.92 \\
\hline $30^{\circ} \mathrm{C}$ & 1.8 & 13.75 \\
\hline $50^{\circ} \mathrm{C}$ & 8.0 & 13,10 \\
\hline $70^{\circ} \mathrm{C}$ & 21.2 & 12.67 \\
\hline $80^{\circ} \mathrm{C}$ & 35 & 12.46 \\
\hline $100^{\circ} \mathrm{C}$ & 73 & 12.14 \\
\hline Sum & & 92,96 \\
\hline
\end{tabular}

etc.

From the above examples we see that the dissociation constant of water determine the programmatic cyber laws. Here are some examples:

\section{Example 1.}

\begin{tabular}{|c|c|}
\hline Water temperature & $\mathrm{K}_{\mathrm{w}} / 10^{-14}$ \\
\hline $80^{\circ} \mathrm{C}$ & 35 \\
\hline Sum & $\mathbf{3 5}$ \\
\hline
\end{tabular}




\begin{tabular}{|c|c|}
\hline Water temperature & $\mathrm{Kw} / 10^{-14}$ \\
\hline $25^{\circ} \mathrm{C}$ & 1.2 \\
\hline $60^{\circ} \mathrm{C}$ & 12.6 \\
\hline $70^{\circ} \mathrm{C}$ & 21.2 \\
\hline Sum & 35 \\
\hline
\end{tabular}

Example 2.

\begin{tabular}{|c|c|c|}
\hline Water temperature & $\mathrm{K}_{\mathrm{w}} / 10^{-14}$ & $\mathrm{pK}_{\mathrm{w}}$ \\
\hline $0^{\circ} \mathrm{C}$ & 0.1 & 14.92 \\
\hline $100^{\circ} \mathrm{C}$ & 73 & 12.14 \\
\hline & $\downarrow$ &
\end{tabular}

\section{Determinate}

\begin{tabular}{|c||c|}
\hline 14,92 & 0 \\
\hline 100 & 12.14 \\
\hline
\end{tabular}

$\operatorname{DET}(14,92,0,100,12,14)=\mathbf{1 4 0 0}$;

\section{Example 3.}

\begin{tabular}{|c|c|c|}
\hline Water temperature & $\mathrm{K}_{\mathrm{w}} / 10^{-14}$ & $\mathrm{pK}_{\mathrm{w}}$ \\
\hline $10^{\circ} \mathrm{C}$ & 0.3 & 14.52 \\
\hline $90^{\circ} \mathrm{C}$ & 53 & 12.28 \\
\hline & $\downarrow$ &
\end{tabular}

\section{Determinate}

\begin{tabular}{|c||c|}
\hline 14,52 & 10 \\
\hline 90 & 12.28 \\
\hline
\end{tabular}

$\operatorname{DET}(14,52,10,90,12,28)=\mathbf{1 1 4 0}$;

etc.

In this example there is also a mathematical balance between constants $\mathrm{pK}_{\mathrm{w}}$ and water temperature. This balance is one of important quantitative characteristics of all processes in chemistry. 


\subsection{Matrix in the water molecule}

We shall now give some mathematical evidences that will prove that in the chemistry of water there really is programmatic and cybernetic algorithm in which it is „recorded“, in the language of mathematics, how the molecule will be built and what will be the quantitative characteristics of the given chemical information.

\begin{tabular}{|c|c|}
\hline Water temperature & $\mathbf{K}_{\mathbf{w}}$ \\
\hline $0{ }^{\circ} \mathrm{C}$ & 0.1 \\
\hline $10^{\circ} \mathrm{C}$ & 0.3 \\
\hline $18^{\circ} \mathrm{C}$ & 0.7 \\
\hline $25^{\circ} \mathrm{C}$ & 1.2 \\
\hline $30^{\circ} \mathrm{C}$ & 1.8 \\
\hline $50^{\circ} \mathrm{C}$ & 8.0 \\
\hline $60^{\circ} \mathrm{C}$ & 12.6 \\
\hline $70^{\circ} \mathrm{C}$ & 21.2 \\
\hline $80^{\circ} \mathrm{C}$ & 35 \\
\hline $90^{\circ} \mathrm{C}$ & 53 \\
\hline $100^{\circ} \mathrm{C}$ & 73 \\
\hline
\end{tabular}

This table contains an overview of constants $\mathbf{K}_{\mathbf{w}}$ The values show some of the quantitative characteristics of the molecule of water. Actually, they show that there is an exact mathematical balance between these constants. Examples:

\begin{tabular}{cccccccccccc}
\multicolumn{10}{c}{ Cumulative } \\
$\mathrm{K}_{\mathrm{w}}$ & $\mathbf{0 , 1}$ & $\mathbf{0 , 3}$ & $\mathbf{0 , 7}$ & $\mathbf{1 , 2}$ & $\mathbf{1 , 8}$ & $\mathbf{8 , 0}$ & $\mathbf{1 2 , 6}$ & $\mathbf{2 1 , 2}$ & $\mathbf{3 5 , 0}$ & $\mathbf{5 3 , 0}$ & $\mathbf{7 3 , 0}$ \\
AP-a & $\mathbf{0 , 1}$ & $\mathbf{0 , 4}$ & $\mathbf{1 , 1}$ & $\mathbf{2 , 3}$ & $\mathbf{4 , 1}$ & $\mathbf{1 2 , 1}$ & $\mathbf{2 4 , 7}$ & $\mathbf{4 5 , 9}$ & $\mathbf{8 0 , 9}$ & $\mathbf{1 3 3 , 9}$ & $\mathbf{2 0 6 , 9}$ \\
AP-b & 206,9 & $\mathbf{2 0 6 , 8}$ & $\mathbf{2 0 6 , 5}$ & $\mathbf{2 0 5 , 8}$ & $\mathbf{2 0 4 , 6}$ & $\mathbf{2 0 2 , 8}$ & $\mathbf{1 9 4 , 8}$ & $\mathbf{1 8 2 , 2}$ & $\mathbf{1 6 1 , 0}$ & $\mathbf{1 2 6 , 0}$ & $\mathbf{7 3 , 0}$
\end{tabular}

$$
(0,1+0,3)=0,4 ;(0,4+0,7)=1,1 ;(1,1+1,2)=2,3 \text {; }
$$

etc.

\begin{tabular}{cccccccccccc}
\multicolumn{10}{c}{ Matrix AP-a } \\
206,9 & 206,8 & 206,5 & $\mathbf{2 0 5 , 8}$ & $\mathbf{2 0 4 , 6}$ & $\mathbf{2 0 2 , 8}$ & $\mathbf{1 9 4 , 8}$ & $\mathbf{1 8 2 , 2}$ & $\mathbf{1 6 1 , 0}$ & $\mathbf{1 2 6 , 0}$ & $\mathbf{7 3 , 0}$ \\
133,9 & $\mathbf{0}$ & $\mathbf{1 3 3 , 8}$ & $\mathbf{1 3 3 , 5}$ & $\mathbf{1 3 2 , 8}$ & $\mathbf{1 3 1 , 6}$ & $\mathbf{1 2 9 , 8}$ & $\mathbf{1 2 1 , 8}$ & $\mathbf{1 0 9 , 2}$ & $\mathbf{8 8 , 0}$ & $\mathbf{5 3 , 0}$ \\
$\mathbf{8 0 , 9}$ & $\mathbf{0}$ & $\mathbf{0}$ & $\mathbf{8 0 , 8}$ & $\mathbf{8 0 , 5}$ & $\mathbf{7 9 , 8}$ & $\mathbf{7 8 , 6}$ & $\mathbf{7 6 , 8}$ & $\mathbf{6 8 , 8}$ & $\mathbf{5 6 , 2}$ & $\mathbf{3 5 , 0}$ \\
$\mathbf{4 5 , 9}$ & $\mathbf{0}$ & $\mathbf{0}$ & $\mathbf{0}$ & $\mathbf{4 5 , 8}$ & $\mathbf{4 5 , 5}$ & $\mathbf{4 4 , 8}$ & $\mathbf{4 3 , 6}$ & $\mathbf{4 1 , 8}$ & $\mathbf{3 3 , 8}$ & $\mathbf{2 1 , 2}$ \\
24,7 & $\mathbf{0}$ & $\mathbf{0}$ & $\mathbf{0}$ & $\mathbf{0}$ & $\mathbf{2 4 , 6}$ & $\mathbf{2 4 , 3}$ & $\mathbf{2 3 , 6}$ & $\mathbf{2 2 , 4}$ & $\mathbf{2 0 , 6}$ & $\mathbf{1 2 , 6}$ \\
12,1 & $\mathbf{0}$ & $\mathbf{0}$ & $\mathbf{0}$ & $\mathbf{0}$ & $\mathbf{0}$ & $\mathbf{1 2 , 0}$ & $\mathbf{1 1 , 7}$ & $\mathbf{1 1 , 0}$ & $\mathbf{9 , 8}$ & $\mathbf{8 , 0}$ \\
4,1 & $\mathbf{0}$ & $\mathbf{0}$ & $\mathbf{0}$ & $\mathbf{0}$ & $\mathbf{0}$ & $\mathbf{0}$ & $\mathbf{4 , 0}$ & $\mathbf{3 , 7}$ & $\mathbf{3 , 0}$ & $\mathbf{1 , 8}$ \\
2,3 & $\mathbf{0}$ & $\mathbf{0}$ & $\mathbf{0}$ & $\mathbf{0}$ & $\mathbf{0}$ & $\mathbf{0}$ & $\mathbf{0}$ & $\mathbf{2 , 2}$ & $\mathbf{1 , 9}$ & $\mathbf{1 , 2}$ \\
1,1 & $\mathbf{0}$ & $\mathbf{0}$ & $\mathbf{0}$ & $\mathbf{0}$ & $\mathbf{0}$ & $\mathbf{0}$ & $\mathbf{0}$ & $\mathbf{0}$ & $\mathbf{1 , 0}$ & $\mathbf{0 , 7}$ \\
$\mathbf{0 , 4}$ & $\mathbf{0}$ & $\mathbf{0}$ & $\mathbf{0}$ & $\mathbf{0}$ & $\mathbf{0}$ & $\mathbf{0}$ & $\mathbf{0}$ & $\mathbf{0}$ & $\mathbf{0}$ & $\mathbf{0 , 3}$ \\
$\mathbf{0 , 1}$ & $\mathbf{0}$ & $\mathbf{0}$ & $\mathbf{0}$ & $\mathbf{0}$ & $\mathbf{0}$ & $\mathbf{0}$ & $\mathbf{0}$ & $\mathbf{0}$ & $\mathbf{0}$ & $\mathbf{0}$
\end{tabular}

$\begin{array}{llllllllll}206,8 & 340,3 & 420,1 & 463,7 & 484,3 & 484,3 & 463,7 & 420,1 & 340,3 & 206,8\end{array}$ $(206,9-0,1)=206,8 ; \quad(206,9-0,4)=206,5 ; \quad ;(206,9-1,1)=205,8 ;$

etc. 
This is one of the digital image water molecules. This picture reveals the secret of digital connection between water temperature and constant $\mathbf{K}_{\mathbf{w}}$ Also, he reveals a balance of mathematical constants. This balance has emerged as a result of chemical processes. It is a result of software, information and cyber laws. Cumulative from the Matrix AP-a are in correlation with each other.

Matrix inversion AP-a.

\begin{tabular}{rlcccccccccc}
206,9 & 0 & 0 & 0 & 0 & 0 & 0 & 0 & 0 & 0 & 0 & 0 \\
133,9 & 0 & $-73,0$ & 0 & 0 & 0 & 0 & 0 & 0 & 0 & 0 & 0 \\
80,9 & 0 & $-53,0$ & $-126,0$ & 0 & 0 & 0 & 0 & 0 & 0 & 0 & 0 \\
45,9 & 0 & $-35,0$ & $-88,0$ & $-161,0$ & 0 & 0 & 0 & 0 & 0 & 0 & 0 \\
24,7 & 0 & $-21,2$ & $-56,2$ & $-109,2$ & $-182,2$ & 0 & 0 & 0 & 0 & 0 & 0 \\
12,1 & 0 & $-12,6$ & $-33,8$ & $-68,8$ & $-121,8$ & $-194,8$ & 0 & 0 & 0 & 0 & 0 \\
4,1 & 0 & $-8,0$ & $-20,6$ & $-41,8$ & $-76,8$ & $-129,8$ & $-202,8$ & 0 & 0 & 0 & 0 \\
2,3 & 0 & $-1,8$ & $-9,8$ & $-22,4$ & $-43,6$ & $-78,6$ & $-131,6$ & $-204,6$ & 0 & 0 & 0 \\
1,1 & 0 & $-1,2$ & $-3,0$ & $-11,0$ & $-23,6$ & $-44,8$ & $-79,8$ & $-132,8$ & $-205,8$ & 0 & 0 \\
0,4 & 0 & $-0,7$ & $-1,9$ & $-3,7$ & $-11,7$ & $-24,3$ & $-45,5$ & $-80,5$ & $-133,5$ & $-206,5$ & 0 \\
0,1 & 0 & $-0,3$ & $-1,0$ & $-2,2$ & $-4,0$ & $-12,0$ & $-24,6$ & $-45,8$ & $-80,8$ & $-133,8$ & $-206,8$ \\
\hline Sum & & $-206,8$ & $-340,3$ & $-420,1$ & $-463,7$ & $-484,3$ & $-484,3$ & $-463,7$ & $-420,1$ & $-340,3$ & $-206,8$
\end{tabular}

Inversion matrix AP is analogous to the matrix of the matrix AP-a. Her task is discreet in its memory keeps information from this matrix.

Matrix AP-b

$\begin{array}{cccccccccccc}206,9 & 133,9 & \mathbf{8 0 , 9} & 45,9 & 24,7 & 12,1 & 4,1 & 2,3 & 1,1 & 0,4 & 0,1 \\ 206,8 & 0 & 133,8 & \mathbf{8 0 , 8} & 45,8 & 24,6 & 12,0 & 4,0 & 2,2 & 1,0 & 0,3 \\ 206,5 & 0 & 0 & 133,5 & 80,5 & 45,5 & 24,3 & 11,7 & 3,7 & 1,9 & 0,7 \\ 205,8 & 0 & 0 & 0 & 132,8 & 79,8 & 44,8 & 23,6 & 11,0 & 3,0 & 1,2 \\ 204,6 & 0 & 0 & 0 & 0 & 131,6 & 78,6 & 43,6 & 22,4 & 9,8 & 1,8 \\ 202,8 & 0 & 0 & 0 & 0 & 0 & 129,8 & 76,8 & 41,8 & 20,6 & \mathbf{8 , 0} \\ 194,8 & 0 & 0 & 0 & 0 & 0 & 0 & 121,8 & \mathbf{6 8 , 8} & 33,8 & 12,6 \\ 182,2 & 0 & 0 & 0 & 0 & 0 & 0 & 0 & 109,2 & 56,2 & 21,2 \\ 161,0 & 0 & 0 & 0 & 0 & 0 & 0 & 0 & 0 & \mathbf{8 8 , 0} & 35,0 \\ 126,0 & 0 & 0 & 0 & 0 & 0 & 0 & 0 & 0 & 0 & 53,0 \\ 73,0 & 0 & 0 & 0 & 0 & 0 & 0 & 0 & 0 & 0 & 0\end{array}$

In this matrix are stored information about the constants $\mathbf{K}_{\mathbf{w}}$. The matrices AP- a and AP-b was determined by the constant $\mathrm{Kw}$, must have water temperatures in this example. 


\section{Matrix inversion AP-b.}

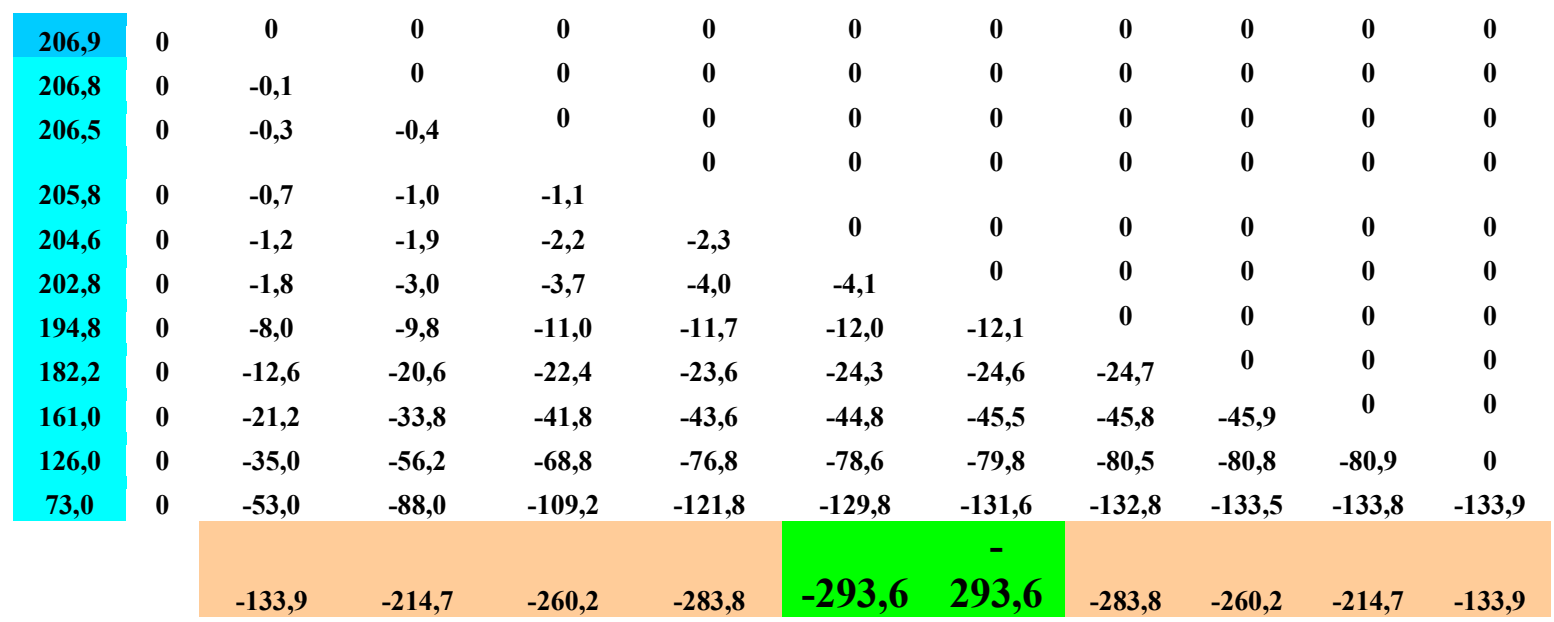

Differences in the mean number totals matrices AP and AP-B associated with the 1907. This code connects the molecules in nature.

$$
[(484,3-293,6) \times 100]=1907 \text {; }
$$

\section{Constant $\mathrm{pK}_{\mathrm{w}}$}

\begin{tabular}{|c|c|}
\hline Water temperature & $\mathrm{pK}_{\mathrm{w}}$ \\
\hline $0^{\circ} \mathrm{C}$ & 14.92 \\
\hline $10^{\circ} \mathrm{C}$ & 14.52 \\
\hline $18^{\circ} \mathrm{C}$ & 14.16 \\
\hline $25^{\circ} \mathrm{C}$ & 13.92 \\
\hline $30{ }^{\circ} \mathrm{C}$ & 13.75 \\
\hline $50{ }^{\circ} \mathrm{C}$ & 13,10 \\
\hline $60^{\circ} \mathrm{C}$ & 12.90 \\
\hline $70^{\circ} \mathrm{C}$ & 12.67 \\
\hline $80^{\circ} \mathrm{C}$ & 12.46 \\
\hline $90^{\circ} \mathrm{C}$ & 12.28 \\
\hline $100^{\circ} \mathrm{C}$ & 12.14 \\
\hline
\end{tabular}

This table contains an overview of constant $\mathrm{pK}_{\mathrm{w}}$. The values show some of the quantitative characteristics of the molecule of water. Actually, they show that there is an exact mathematical balance between these constants. Examples: 


\section{Cumulative}

$\begin{array}{cccccccccccc} & 12,14 & 12,28 & 12,46 & 12,67 & 12,90 & 13,10 & 13,75 & 13,92 & 14,16 & 14,52 & 14,92 \\ \text { APa } & 12,14 & 24,42 & 36,88 & 49,55 & 62,45 & 75,55 & \mathbf{8 9 , 3 0} & 103,22 & 117,38 & 131,90 & 146,82 \\ \text { APb } & 146,82 & 134,68 & 122,40 & 109,94 & \mathbf{9 7 , 2 7} & \mathbf{8 4 , 3 7} & \mathbf{7 1 , 2 7} & \mathbf{5 7 , 5 2} & \mathbf{4 3 , 6 0} & \mathbf{2 9 , 4 4} & \mathbf{1 4 , 9 2}\end{array}$

Matrix AP-a

$\begin{array}{cccccccccccc}\mathbf{1 4 6 , 8 2} & \mathbf{1 3 4 , 6 8} & \mathbf{1 2 2 , 4 0} & \mathbf{1 0 9 , 9 4} & \mathbf{9 7 , 2 7} & \mathbf{8 4 , 3 7} & \mathbf{7 1 , 2 7} & \mathbf{5 7 , 5 2} & \mathbf{4 3 , 6 0} & \mathbf{2 9 , 4 4} & \mathbf{1 4 , 9 2} \\ 131,90 & \mathbf{0} & \mathbf{1 1 9 , 7 6} & \mathbf{1 0 7 , 4 8} & \mathbf{9 5 , 0 2} & \mathbf{8 2 , 3 5} & \mathbf{6 9 , 4 5} & \mathbf{5 6 , 3 5} & \mathbf{4 2 , 6 0} & \mathbf{2 8 , 6 8} & \mathbf{1 4 , 5 2} \\ \mathbf{1 1 7 , 3 8} & \mathbf{0} & \mathbf{0} & \mathbf{1 0 5 , 2 4} & \mathbf{9 2 , 9 6} & \mathbf{8 0 , 5 0} & \mathbf{6 7 , 8 3} & \mathbf{5 4 , 9 3} & \mathbf{4 1 , 8 3} & \mathbf{2 8 , 0 8} & \mathbf{1 4 , 1 6} \\ \mathbf{1 0 3 , 2 2} & \mathbf{0} & \mathbf{0} & \mathbf{0} & \mathbf{9 1 , 0 8} & \mathbf{7 8 , 8 0} & \mathbf{6 6 , 3 4} & \mathbf{5 3 , 6 7} & \mathbf{4 0 , 7 7} & \mathbf{2 7 , 6 7} & \mathbf{1 3 , 9 2} \\ \mathbf{8 9 , 3 0} & \mathbf{0} & \mathbf{0} & \mathbf{0} & \mathbf{0} & \mathbf{7 7 , 1 6} & \mathbf{6 4 , 8 8} & \mathbf{5 2 , 4 2} & \mathbf{3 9 , 7 5} & \mathbf{2 6 , 8 5} & \mathbf{1 3 , 7 5} \\ \mathbf{7 5 , 5 5} & \mathbf{0} & \mathbf{0} & \mathbf{0} & \mathbf{0} & \mathbf{0} & \mathbf{6 3 , 4 1} & \mathbf{5 1 , 1 3} & \mathbf{3 8 , 6 7} & \mathbf{2 6 , 0 0} & \mathbf{1 3 , 1 0} \\ \mathbf{6 2 , 4 5} & \mathbf{0} & \mathbf{0} & \mathbf{0} & \mathbf{0} & \mathbf{0} & \mathbf{0} & \mathbf{5 0 , 3 1} & \mathbf{3 8 , 0 3} & \mathbf{2 5 , 5 7} & \mathbf{1 2 , 9 0} \\ \mathbf{4 9 , 5 5} & \mathbf{0} & \mathbf{0} & \mathbf{0} & \mathbf{0} & \mathbf{0} & \mathbf{0} & \mathbf{0} & \mathbf{3 7 , 4 1} & \mathbf{2 5 , 1 3} & \mathbf{1 2 , 6 7} \\ \mathbf{3 6 , 8 8} & \mathbf{0} & \mathbf{0} & \mathbf{0} & \mathbf{0} & \mathbf{0} & \mathbf{0} & \mathbf{0} & \mathbf{0} & \mathbf{2 4 , 7 4} & \mathbf{1 2 , 4 6} \\ \mathbf{2 4 , 4 2} & \mathbf{0} & \mathbf{0} & \mathbf{0} & \mathbf{0} & \mathbf{0} & \mathbf{0} & \mathbf{0} & \mathbf{0} & \mathbf{0} & \mathbf{1 2 , 2 8} \\ 12,14 & \mathbf{0} & \mathbf{0} & \mathbf{0} & \mathbf{0} & \mathbf{0} & \mathbf{0} & \mathbf{0} & \mathbf{0} & \mathbf{0} & \mathbf{0}\end{array}$

Matrix inversion AP-a

$\begin{array}{cccccccccccc}146,82 & 0 & 0 & 0 & 0 & 0 & 0 & 0 & 0 & 0 & 0 & 0 \\ 131,90 & 0 & -14,92 & 0 & 0 & 0 & 0 & 0 & 0 & 0 & 0 & 0 \\ 117,38 & 0 & -14,52 & -29,44 & 0 & 0 & 0 & 0 & 0 & 0 & 0 & 0 \\ 103,22 & 0 & -14,16 & -28,68 & -43,60 & 0 & 0 & 0 & 0 & 0 & 0 & 0 \\ 89,30 & 0 & -13,92 & -28,08 & -42,60 & -57,52 & 0 & 0 & 0 & 0 & 0 & 0 \\ 75,55 & 0 & -13,75 & -27,67 & -41,83 & -56,35 & -71,27 & 0 & 0 & 0 & 0 & 0 \\ 62,45 & 0 & -13,10 & -26,85 & -40,77 & -54,93 & -69,45 & -84,37 & 0 & 0 & 0 & 0 \\ 49,55 & 0 & -12,90 & -26,00 & -39,75 & -53,67 & -67,83 & -82,35 & -97,27 & 0 & 0 & 0 \\ 36,88 & 0 & -12,67 & -25,57 & -38,67 & -52,42 & -66,34 & -80,50 & -95,02 & -109,94 & 0 & 0 \\ 24,42 & 0 & -12,46 & -25,13 & -38,03 & -51,13 & -64,88 & -78,80 & -92,96 & -107,48 & -122,40 & 0 \\ 12,14 & 0 & -12,28 & -24,74 & -37,41 & -50,31 & -63,41 & -77,16 & -91,08 & -105,24 & -119,76 & -134,68 \\ \text { Sum } & & -134,68 & -242,16 & -322,66 & -376,33 & -403,18 & -403,18 & -376,33 & -322,66 & -242,16 & -134,68\end{array}$

Matrix AP-b

\begin{tabular}{|c|c|c|c|c|c|c|c|c|c|c|}
\hline 146,82 & 131,90 & 117,38 & 103,22 & 89,30 & 75,55 & 62,45 & 49,55 & 36,88 & 24,42 & 12,14 \\
\hline 134,68 & 0 & 119,76 & 105,24 & 91,08 & 77,16 & 63,41 & 50,31 & 37,41 & 24,74 & 12,28 \\
\hline 122,40 & 0 & 0 & 107,48 & 92,96 & 78,80 & 64,88 & 51,13 & 38,03 & 25,13 & 12,46 \\
\hline 10994 & 0 & $\mathbf{0}$ & 0 & 95,02 & 80,50 & 66,34 & 52,42 & 38,67 & 25,57 & 12,67 \\
\hline 97,27 & 0 & 0 & 0 & 0 & 82,35 & 67,83 & 53,67 & 39,75 & 26,00 & 12,90 \\
\hline 84,37 & 0 & 0 & 0 & 0 & $\mathbf{0}$ & 69,45 & 54,93 & 40,77 & 26,85 & 13,10 \\
\hline 71,27 & 0 & 0 & $\mathbf{0}$ & 0 & $\mathbf{0}$ & 0 & 56,35 & 41,83 & 27,67 & 13,75 \\
\hline 57,52 & 0 & 0 & 0 & 0 & 0 & 0 & 0 & 42,60 & 28,08 & 13,92 \\
\hline 43,60 & 0 & 0 & 0 & 0 & $\mathbf{0}$ & 0 & $\mathbf{0}$ & 0 & 28,68 & 14,16 \\
\hline 29,44 & 0 & 0 & $\mathbf{0}$ & 0 & $\mathbf{0}$ & 0 & $\mathbf{0}$ & 0 & $\mathbf{0}$ & 14,52 \\
\hline 14,92 & $\mathbf{0}$ & $\mathbf{0}$ & $\mathbf{0}$ & $\mathbf{0}$ & $\mathbf{0}$ & $\mathbf{0}$ & $\mathbf{0}$ & $\mathbf{0}$ & o & 0 \\
\hline Sum & 131,90 & 237,14 & 315,94 & 368,36 & 394,36 & 394,36 & 368,36 & 315,94 & 237,14 & 131,90 \\
\hline
\end{tabular}




\section{Matrix inversion AP-b}

\begin{tabular}{|c|c|c|c|c|c|c|c|c|c|c|c|}
\hline 146,82 & 0 & $\mathbf{0}$ & 0 & $\mathbf{0}$ & $\mathbf{0}$ & 0 & 0 & 0 & 0 & 0 & 0 \\
\hline 134,68 & 0 & $-12,14$ & 0 & $\mathbf{0}$ & $\mathbf{0}$ & 0 & 0 & $\mathbf{0}$ & 0 & 0 & 0 \\
\hline 122,40 & 0 & $-12,28$ & $-24,42$ & 0 & 0 & 0 & 0 & $\mathbf{0}$ & 0 & 0 & $\mathbf{0}$ \\
\hline 109,94 & $\mathbf{0}$ & $-12,46$ & $-24,74$ & $-36,88$ & 0 & 0 & 0 & 0 & 0 & $\mathbf{0}$ & $\mathbf{0}$ \\
\hline 97,27 & 0 & $-12,67$ & $-25,13$ & $-37,41$ & $-49,55$ & 0 & 0 & 0 & 0 & 0 & $\mathbf{0}$ \\
\hline 84,37 & 0 & $-12,90$ & $-25,57$ & $-38,03$ & $-50,31$ & $-62,45$ & $\mathbf{0}$ & 0 & 0 & $\mathbf{0}$ & $\mathbf{0}$ \\
\hline 71,27 & $\mathbf{0}$ & $-13,10$ & $-26,00$ & $-38,67$ & $-51,13$ & $-63,41$ & $-75,55$ & 0 & $\mathbf{0}$ & $\mathbf{0}$ & $\mathbf{0}$ \\
\hline $\mathbf{5 7 , 5 2}$ & 0 & $-13,75$ & $-26,85$ & $-39,75$ & $-52,42$ & $-64,88$ & $-77,16$ & $-89,30$ & 0 & 0 & $\mathbf{0}$ \\
\hline 43,60 & $\mathbf{0}$ & $-13,92$ & $-27,67$ & $-40,77$ & $-53,67$ & $-66,34$ & $-78,80$ & $-91,08$ & $-103,22$ & $\mathbf{0}$ & $\mathbf{0}$ \\
\hline 29,44 & 0 & $-14,16$ & $-28,08$ & $-41,83$ & $-54,93$ & $-67,83$ & $\mathbf{- 8 0 , 5 0}$ & $-92,96$ & $-105,24$ & $-117,38$ & 0 \\
\hline 14,92 & 0 & $-14,52$ & $-28,68$ & $-42,60$ & $-56,35$ & $-69,45$ & $-82,35$ & $-95,02$ & $-107,48$ & $-119,76$ & $-131,90$ \\
\hline Sum & & $-131,90$ & $-237,14$ & $-315,94$ & $-368,36$ & $-394,36$ & $-394,36$ & $-368,36$ & $-315,94$ & $-237,14$ & $-131,90$ \\
\hline
\end{tabular}

Now we have the answer to the question: Why hot water freezes faster than cold? The answer is as follows: Cybernetic information principles in your digital program to determine the hot water freezes faster than cold water. It is written in the nature of the algorithm. Nature has decided that this is so. Is it really so testify digital image molecule of water. These pictures have countless. We mentioned this in just a few of these images. We hope that researchers in the field of chemistry will soon decode and other digital images.

\section{4. Chemical equilibrium}

Under the law of mass action of reaction product is proportional to the concentration of substances. Under the law of mass action of reaction product is proportional to the concentration of substances. At temperatures of 60-10000 hydrogen and oxygen to combine with the explosion, and produced water, and water temperatures of 4000-50000 with an explosion breaks down into hydrogen and oxygen.

The reaction between hydrogen and oxygen is reversible.

$$
2 \mathrm{H}_{2}+\mathrm{O}_{2}=2 \mathrm{H}_{2} \mathrm{O}
$$

In this way, chemical equilibrium is established. So, is done and the creation of water molecules and their decomposition. Numerical value of $\mathrm{K}$ determines the position of equilibrium at a given temperature. Therefore, the constant $\mathrm{K}$ preheated water has a numerical value, and the other with hot water, so the warmer water freezes faster than in the preheated. In this way the rapid freezing of water establishes the position of equilibrium hydrogen and oxygen at a given temperature. The equilibrium constant is calculated as follows:

$$
\begin{gathered}
\mathrm{K} 1=\left[\mathrm{H}_{2} \mathrm{O}\right]^{2} \\
\mathrm{~K} 2=\left[\mathrm{H}_{2}\right]^{2}\left[\mathrm{O}_{2}\right] \\
\mathrm{mn}(\mathrm{K} 1: \mathrm{K} 2)=\mathrm{K}
\end{gathered}
$$


The principle of moving equilibrium (Lešatelje, 1884): If the system that is in balance, made apoljni impacts, this balance shifts in the direction of influence and so long as the opposite effect, which is more and more increases in the system, not to equalize with the outside influence. Therefore, warm water freezes faster than preheated.

The equation shows that $2 \mathrm{H}_{2}+\mathrm{O}_{2}=2 \mathrm{H}_{2} \mathrm{O}+137 \mathrm{kcal}$ is incorporating hydrogen with oxygen followed by separation of heat and the decomposition of water by absorbing heat. If more heat is brought into the system, so much more beneficial effect on the decomposition of water, is the endothermic reaction.

\section{CONCLUSION}

Conversely, withdrawal of heat by cooling complicate the decomposition of water (exothermic reaction). If this system at a certain temperature is in equilibrium, and then warms up, the balance will be appropriately moved to the side creating a larger concentration of free hydrogen and oxygen.

So, when heating a balanced system, and this water is preheated, the balance shifts to the side of endothermic reactions, and on cooling the side exothermic. Thus, hot water freezes faster than preheated to establish a balance of hydrogen and oxygen at a given temperature. The equation shows that $\mathrm{X}$ is incorporating hydrogen with oxygen followed by separation of heat and the decomposition of water by absorbing heat.

\section{References}

[1] K. C. Chou, Gene Cloning \& Expression Technologies, Chapter 4 (Weinrer, P. W.)

[2] L. Kurić, International Letters of Chemistry, Physics and Astronomy 10 (2014) 62-73.

[3] L. Kurić, J. Comput. Sci. Biol. 2 (2009) 101-116.

[4] L. Kurić, Journal de la Societe de statistique de Paris 127(2) (1986).

[5] L. Kurić, The Insulin Bio Code - Zero Frenquencies, GJMR 10(1) (2010) 15.

[6] L. Kurić, Advances and Applications in Bioinformatics and Chemistry, 2010, 45-58.

[7] L. Kurić, GJMR 1(1) (2010) 15.

[8] L. Kurić, International Journal of Computer Technology and Application 2(2) (2011) 216-241.

[9] L. Kurić, International Journal of Computer Technology and Application 2(2) (2011) 258-273.

[10] L. Kurić, Journal of Chemical Enginerring and Material Science 2(5) (2011).

[11] L. Kurić, International Letters of Chemistry, Physics and Astronomy 11(3) (2014) 202-213. 
[12] L. Kurić, International Letters of Chemistry, Physics and Astronomy 12 (2014) 31-50.

[13] L. Kurić, International Letters of Chemistry, Physics and Astronomy 13(1) (2014) 11-20.

[14] L. Kurić, International Letters of Chemistry, Physics and Astronomy 13(1) (2014) 42-53. 